\title{
A Priori Estimates and Continuous Dependence of Solutions of Mixed Problems for Parabolic Equations As Nonlocal Boundary Conditions Pass into Local Ones
}

\author{
N. I. Yurchuk and Charie Koku \\ Belarus State University, Minsk, Belarus \\ Received May 18, 2007
}

Dedicated to the sixtieth birthday of Academician E.I. Moiseev

It is well known that, for $\alpha=0$ and $\alpha=1$, the corresponding problems with local conditions are solvable, and the solutions are unique and belong to $W_{2}^{2,1}(G)$.

We prove the existence and uniqueness of solutions of the family of problems with nonlocal conditions for each $\alpha \in(0,1)$. For the differences $u_{\alpha}-u_{0}$ and $u_{\alpha}-u_{1}(0<\alpha<1)$, we establish a priori estimates and use them to prove that if $\varphi_{\alpha} \rightarrow \varphi_{0}$ as $\alpha \rightarrow 0$, then $u_{\alpha} \rightarrow u_{0}$ and if $\varphi_{\alpha} \rightarrow \varphi_{1}$ as $\alpha \rightarrow 1$, then $u_{\alpha} \rightarrow u_{1}$.

DOI: $10.1134 /$ S0012266108030142

\section{STATEMENT OF THE PROBLEM}

In the rectangle $G=(0,1) \times(0, T)$, we consider the family of mixed problems with nonlocal conditions

$$
\begin{gathered}
\frac{1}{a(x, t)} \frac{\partial u_{\alpha}}{\partial t}-\frac{\partial^{2} u_{\alpha}}{\partial x^{2}}=f(x, t), \quad 0<\alpha<1, \\
u_{\alpha}(x, 0)=\varphi_{\alpha}(x), \quad u_{\alpha}(0, t)=0, \quad \frac{u_{\alpha}(1, t)-u_{\alpha}(\alpha, t)}{1-\alpha}=h(t)
\end{gathered}
$$

and two mixed problems with local conditions:

$$
\begin{array}{rlrl}
\frac{1}{a(x, t)} \frac{\partial u_{0}}{\partial t}-\frac{\partial^{2} u_{0}}{\partial x^{2}} & =f(x, t), \\
u_{0}(x, 0)=\varphi_{0}(x), \quad & u_{0}(0, t)=0, \quad u_{0}(1, t) & =h(t), \\
\frac{1}{a(x, t)} \frac{\partial u_{1}}{\partial t}-\frac{\partial^{2} u_{1}}{\partial x^{2}} & =f(x, t), \\
u_{1}(x, 0)=\varphi_{1}(x), & u_{1}(0, t)=0, & \frac{\partial u_{1}(1, t)}{\partial x} & =h(t) .
\end{array}
$$


We assume that the coefficient $a(x, t)$ in Eqs. (1), (3), and (5) is a continuously differentiable function and

$$
\begin{aligned}
a_{1} & \geq a(x, t) \geq a_{0}>0, \quad f \in L_{2}(G), \quad h \in W_{2}^{1}(0, T), \\
\varphi_{\alpha}, \varphi_{0}, \varphi_{1} & \in W_{2}^{1}(0,1), \quad \varphi_{\alpha}(0)=\varphi_{0}(0)=\varphi_{1}(0)=0, \\
\varphi_{0}(1) & =h(0), \quad \varphi_{1}^{\prime}(1)=h(0), \quad \frac{\varphi_{\alpha}(1)-\varphi_{\alpha}(\alpha)}{1-\alpha}=h(0) .
\end{aligned}
$$

It is known that if these assumptions hold, then there exist unique solutions of problem (3), (4) and (5), (6), and these solutions have first derivatives with respect to $t$ and derivatives of order $\leq 2$ with respect to $x$ almost everywhere in $G$. If the problem data are smoother, then so are the solutions. In the present paper, we prove the existence and uniqueness of solutions of the family of problems (1), (2) for each $0<\alpha<1$.

Problem (3), (4) is the limit of the family of problems (1), (2) as $\alpha \rightarrow 0$, and problem (5), (6) is the limit of the family of problems (1), (2) as $\alpha \rightarrow 1$.

In the present paper, we prove a priori estimates for the differences $u_{\alpha}-u_{0}$ and $u_{\alpha}-u_{1}$ and use them to show that if $\varphi_{\alpha} \rightarrow \varphi_{0}$ as $\alpha \rightarrow 0$, then $u_{\alpha} \rightarrow u_{0}$ and if $\varphi_{\alpha} \rightarrow \varphi_{1}$ as $\alpha \rightarrow 1$, then $u_{\alpha} \rightarrow u_{1}$. Thus, we establish the following new important property: the solutions of mixed problems for parabolic equations with nonlocal conditions in (2) change continuously as these conditions pass into the local ones.

Note that a similar property was obtained in [1] for the mixed problems (1), (2) for the case in which the nonlocal condition in (2) is replaced by the nonlocal integral condition

$$
\frac{1}{\alpha} \int_{1-\alpha}^{1} u_{\alpha}(x, t) d x=h(t)
$$

and $u_{\alpha} \rightarrow u_{0}$ as $\alpha \rightarrow 0$, where $u_{0}$ is the solution of problem (3), (4). Problems with a condition of the form (7) were earlier studied in $[2-7]$.

\section{A PRIORI ESTIMATES AND THE EXISTENCE OF GENERALIZED SOLUTIONS OF PROBLEMS (1), (2)}

If we replace the unknown functions in problem (1), (2) by the formula

$$
u_{\alpha}(x, t)=v_{\alpha}(x, t)+x h(t)
$$

then, for the new functions $v_{\alpha}(x, t)$, we obtain the problem

$$
\begin{aligned}
\frac{1}{a(x, t)} \frac{\partial v_{\alpha}}{\partial t}-\frac{\partial^{2} v_{\alpha}}{\partial x^{2}} & =\tilde{f}(x, t) \equiv f(x, t)-\frac{x}{a} h^{\prime}(t), \\
v_{\alpha}(x, 0)=\tilde{\varphi}_{\alpha}(x) & \equiv \varphi_{\alpha}(x)-x h(0), \quad v_{\alpha}(0, t)=0, \quad \frac{v_{\alpha}(1, t)-v_{\alpha}(\alpha, t)}{1-\alpha}=0
\end{aligned}
$$

for each $\alpha \in(0,1)$.

Theorem 1. The solutions $v_{\alpha}$ of problem (9), (10) satisfy the inequality

$$
\begin{aligned}
\sup _{0 \leq t \leq T} \int_{0}^{1} \psi_{\alpha}(x) v_{\alpha}^{2}(x, t) d x+\int_{0}^{T} \int_{0}^{1} \psi_{\alpha}(x)\left(\frac{\partial v_{\alpha}(x, t)}{\partial x}\right)^{2} d x d t \\
\leq C\left(\int_{0}^{1} \psi_{\alpha}(x) \tilde{\varphi}_{\alpha}^{2}(x) d x+\int_{0}^{T} \int_{0}^{1} \psi_{\alpha}(x) \tilde{f}^{2}(x, t) d x d t\right),
\end{aligned}
$$

DIFFERENTIAL EQUATIONS $\quad$ Vol. 44 No. 32008 
where the constant $C$ is independent of $v_{\alpha}$ and

$$
\psi_{\alpha}= \begin{cases}1 & \text { if } 0 \leq x \leq \alpha \\ (1-x) /(1-\alpha) & \text { if } \quad \alpha \leq x \leq 1\end{cases}
$$

Proof. By integrating by parts, we obtain the identities

$$
\begin{aligned}
\int_{0}^{1} \frac{\psi_{\alpha}}{a} \frac{\partial v_{\alpha}}{\partial t} v_{\alpha} d x & =\frac{1}{2} \frac{\partial}{\partial t} \int_{0}^{1} \frac{\psi_{\alpha}}{a} v_{\alpha}^{2} d x-\frac{1}{2} \int_{0}^{1} \frac{\partial}{\partial t}\left(\frac{\psi_{\alpha}}{a}\right) v_{\alpha}^{2} d x \\
-\int_{0}^{1} \psi_{\alpha} \frac{\partial^{2} v_{\alpha}}{\partial x^{2}} v_{\alpha} d x & =-\int_{0}^{\alpha} \frac{\partial^{2} v_{\alpha}}{\partial x^{2}} v_{\alpha} d x-\int_{\alpha}^{1} \frac{1-x}{1-\alpha} \frac{\partial^{2} v_{\alpha}}{\partial x^{2}} v_{\alpha} d x \\
-\int_{0}^{\alpha} \frac{\partial^{2} v_{\alpha}}{\partial x^{2}} v_{\alpha} d x= & \int_{0}^{\alpha}\left(\frac{\partial v_{\alpha}}{\partial x}\right)^{2} d x-\frac{\partial v_{\alpha}(\alpha-0, t)}{\partial x} v_{\alpha}(\alpha-0, t), \\
-\int_{\alpha}^{1} \frac{1-x}{1-\alpha} \frac{\partial^{2} v_{\alpha}}{\partial x^{2}} v_{\alpha} d x= & \int_{\alpha}^{1} \frac{1-x}{1-\alpha}\left(\frac{\partial v_{\alpha}}{\partial x}\right)^{2} d x+\frac{\partial v_{\alpha}(\alpha+0, t)}{\partial x} v_{\alpha}(\alpha+0, t) \\
& -\frac{1}{1-\alpha} \int_{\alpha}^{1} \frac{\partial v_{\alpha}}{\partial x} v_{\alpha} d x \\
2 \int_{\alpha}^{1} \frac{\partial v_{\alpha}}{\partial x} v_{\alpha} d x= & v_{\alpha}^{2}(1, t)-v_{\alpha}^{2}(\alpha, t)=0 .
\end{aligned}
$$

By multiplying both sides of Eq. (9) by $e^{c(\tau-t)} \psi_{\alpha} v_{\alpha}$, by integrating the resulting relation with respect to $x$ from 0 to 1 and with respect to $t$ from 0 to $0<\tau \leq T$, and by using identities (12)-(16), we obtain

$$
\begin{aligned}
\frac{1}{2} \int_{0}^{1} \frac{\psi_{\alpha}(x)}{a(x, \tau)} & v_{\alpha}^{2}(x, \tau) d x+\int_{0}^{\tau} \int_{0}^{1} e^{c(\tau-t)} \psi_{\alpha}(x)\left(\frac{\partial v_{\alpha}(x, \tau)}{\partial x}\right)^{2} d x d t \\
= & \frac{e^{c \tau}}{2} \int_{0}^{1} \frac{\psi_{\alpha}(x)}{a(x, 0)} \tilde{\varphi}_{\alpha}^{2}(x) d x+\int_{0}^{\tau} \int_{0}^{1} e^{c(\tau-t)} \psi_{\alpha}(x) \tilde{f}(x, t) v_{\alpha}(x, t) d x d t \\
& -\frac{1}{2} \int_{0}^{\tau} \int_{0}^{1} e^{c(\tau-t)} \frac{\psi_{\alpha}(x)}{a^{2}(x, t)}\left(\frac{\partial a(x, t)}{\partial t}+c a(x, t)\right) v_{\alpha}^{2}(x, t) d x d t
\end{aligned}
$$

We choose the constant $c$ in (17) so as to ensure that $-\frac{\partial a(x, t)}{\partial t} \leq c a(x, t)$. Then the third term on the right-hand side in (17) is nonpositive, and we omit it. The second term on the right-hand side in (17) can be estimated by the quantity

$$
\frac{\varepsilon}{4} T \sup _{0 \leq t \leq T} \int_{0}^{1} \psi_{\alpha}(x) v_{\alpha}^{2}(x, t) d x+\frac{e^{2 c T}}{\varepsilon} \int_{0}^{T} \int_{0}^{1} \psi_{\alpha}(x) \tilde{f}^{2}(x, t) d x d t
$$


where $\varepsilon>0$ is an arbitrary number. After that, from (17), we obtain the inequality

$$
\begin{aligned}
\frac{1}{2 a_{1}} \int_{0}^{1} \psi_{\alpha}(x) & v_{\alpha}^{2}(x, t) d x+\int_{0}^{\tau} \int_{0}^{1} \psi_{\alpha}(x)\left(\frac{\partial v_{\alpha}(x, t)}{\partial x}\right)^{2} d x d t \\
\leq & \frac{e^{c T}}{2 a_{0}} \int_{0}^{1} \psi_{\alpha}(x) \tilde{\varphi}_{\alpha}^{2}(x) d x+\frac{e^{2 c T}}{\varepsilon} \int_{0}^{T} \int_{0}^{1} \psi_{\alpha}(x) \tilde{f}^{2}(x, t) d x d t \\
& +\frac{\varepsilon}{4} T \sup _{0 \leq t \leq T} \int_{0}^{1} \psi_{\alpha}(x) v_{\alpha}^{2}(x, t) d x,
\end{aligned}
$$

whose right-hand side is independent of $\tau$. Then we take the least upper bound (sup) with respect to $0 \leq \tau \leq T$ on the left-hand side of this inequality and set $\varepsilon=1 /\left(a_{1} T\right)$. As a result, we obtain inequality (11), where

$$
C=e^{c T} \max \left(2 a_{1} / a_{0}, 4 T e^{c T} a_{1}^{2}\right) .
$$

The proof of Theorem 1 is complete.

Let us proceed to the proof of the existence of a strong generalized solution of problem (9), (10). For the solution of problem (9), (10), we introduce the space $E_{\alpha}$ with the norm

$$
\left\|v_{\alpha}\right\|_{E_{\alpha}}^{2}=\sup _{0 \leq t \leq T} \int_{0}^{1} \psi_{\alpha}(x) v_{\alpha}^{2}(x, t) d x+\int_{0}^{T} \int_{0}^{1} \psi_{\alpha}(x)\left(\frac{\partial v_{\alpha}(x, t)}{\partial x}\right)^{2} d x d t
$$

given by the left-hand side of (11). The right-hand side $\tilde{f}$ of Eq. (9) and the initial data $\tilde{\varphi}_{\alpha}$ are considered in the space $F_{\alpha}$ of vector functions $\tilde{F}_{\alpha}=\left(\tilde{\varphi}_{\alpha}, \tilde{f}\right)$ with the norm

$$
\left\|\tilde{F}_{\alpha}\right\|_{F_{\alpha}}^{2}=\int_{0}^{1} \psi_{\alpha}(x) \tilde{\varphi}^{2}(x) d x+\int_{0}^{T} \int_{0}^{1} \psi_{\alpha}(x) \tilde{f}^{2}(x, t) d x d t
$$

given by the right-hand side of inequality (11). Problem (9), (10) corresponds to the operator $L_{\alpha}=\left(\mathcal{L}_{\alpha}, l\right), \mathcal{L}_{\alpha} v_{\alpha} \equiv \frac{1}{a} \frac{\partial v_{\alpha}}{\partial t}-\frac{\partial^{2} v_{\alpha}}{\partial x^{2}}, l v_{\alpha}=v_{\alpha}(x, 0)$, mapping $E_{\alpha}$ into $F_{\alpha}$ with domain

$$
D\left(L_{\alpha}\right)=\left\{v_{\alpha} \in W_{2}^{2,1}(G): v_{\alpha}(0, t)=0, \frac{v_{\alpha}(1, t)-v_{\alpha}(\alpha, t)}{1-\alpha}=0\right\} .
$$

In a standard way, one can show [8, p. 92 of the Russian translation] that the operator $L_{\alpha}: E_{\alpha}^{\rightarrow} F_{\alpha}$ is closable. Its closure will be denoted by $\bar{L}_{\alpha}$, and the domain of $\bar{L}_{\alpha}$ will be denoted by $D\left(\bar{L}_{\alpha}\right)$.

Definition. A solution of the equation $\bar{L}_{\alpha} v_{\alpha}=\tilde{F}_{\alpha} \in F_{\alpha}$ is called a strong generalized solution of problem (9), (10).

In other words, a function $v_{\alpha}$ is called a strong generalized solution of problem (9), (10) if there exists a sequence of functions $v_{\alpha, n} \in D\left(L_{\alpha}\right)$ such that $\left\|v_{\alpha, n}-v_{\alpha}\right\|_{E_{\alpha}} \rightarrow 0$ and $\left\|L_{\alpha} v_{\alpha, n}-\tilde{F}_{\alpha}\right\|_{F_{\alpha}} \rightarrow 0$ as $n \rightarrow \infty$. For the sequences $v_{\alpha, n} \in D\left(L_{\alpha}\right)$, we have the inequalities

$$
\left\|v_{\alpha, n}\right\|_{E_{\alpha}}^{2} \leq C\left\|L_{\alpha} v_{\alpha, n}\right\|_{F_{\alpha}}^{2}
$$

which follow from Theorem 1. By passing to the limit in (19), we obtain the inequality

$$
\left\|v_{\alpha}\right\|_{E_{\alpha}}^{2} \leq C\left\|\bar{L}_{\alpha} v_{\alpha}\right\|_{F_{\alpha}}^{2}
$$

DIFFERENTIAL EQUATIONS $\quad$ Vol. $44 \quad$ No. $3 \quad 2008$ 
which implies that the strong generalized solution of problem (9), (10) is unique and the range of $\bar{L}_{\alpha}$ satisfies $R\left(\bar{L}_{\alpha}\right)=\overline{R\left(L_{\alpha}\right)}$. Therefore, to prove the existence of a strong generalized solution, one should show that $R\left(L_{\alpha}\right)$ is dense in $F_{\alpha}$. In turn, since the range of the trace operator $l$ is dense in $L_{2}(0,1)$ with weight $\psi_{\alpha}$, it suffices to show that the relation

$$
\int_{0}^{T} \int_{0}^{1}\left(\frac{1}{a} \frac{\partial v_{\alpha}}{\partial t}-\frac{\partial^{2} v_{\alpha}}{\partial x^{2}}\right) \psi_{\alpha}(x) g(x, t) d x d t=0
$$

where $v_{\alpha}$ ranges over $D_{0}\left(L_{\alpha}\right)=\left\{v_{\alpha} \in D\left(L_{\alpha}\right): v_{\alpha}(x, 0)=0\right\}$ and $g \in L_{2}(G)$, implies that $g \equiv 0$.

In $(21)$, we set

$$
v_{\alpha}(x, t)=\int_{0}^{t} e^{c(T-\tau)}\left[\int_{0}^{x}(x-\xi) \frac{g(\xi, \tau)}{a(\xi, \tau)} d \xi-x \int_{0}^{1} \psi_{\alpha}(\xi) \frac{g(\xi, \tau)}{a(\xi, \tau)} d \xi\right] d \tau
$$

Then $g(x, t)=a(x, t) \frac{\partial^{3} v_{\alpha}(x, t)}{\partial x^{2} \partial t}$, and from $(21)$, we obtain

$$
\int_{0}^{T} \int_{0}^{1} e^{c(T-t)} \psi_{\alpha}(x)\left(\frac{\partial v_{\alpha}}{\partial t} \frac{\partial^{3} v_{\alpha}}{\partial x^{2} \partial t}-a \frac{\partial^{2} v_{\alpha}}{\partial x^{2}} \frac{\partial^{3} v_{\alpha}}{\partial t \partial x^{2}}\right) d x d t=0
$$

Just as in (12)-(16), we prove the identities

$$
\begin{aligned}
\int_{0}^{T} \int_{0}^{1} e^{c(T-t)} \psi_{\alpha}(x) \frac{\partial v_{\alpha}}{\partial t} \frac{\partial^{3} v_{\alpha}}{\partial x^{2} \partial t} d x d t= & -\int_{0}^{T} \int_{0}^{1} e^{c(T-t)} \psi_{\alpha}(x)\left(\frac{\partial^{2} v_{\alpha}}{\partial x \partial t}\right)^{2} d x d t \\
\int_{0}^{T} \int_{0}^{1} e^{c(T-t)} \psi_{\alpha}(x) a \frac{\partial^{2} v_{\alpha}}{\partial x^{2}} \frac{\partial^{3} v_{\alpha}}{\partial t \partial x^{2}} d x d t= & \left.\int_{0}^{1} \psi_{\alpha}(x) a\left(\frac{\partial^{2} v_{\alpha}}{\partial x^{2}}\right)^{2} d x\right|_{t=T} \\
& +\int_{0}^{T} \int_{0}^{1}\left(a c-\frac{\partial a}{\partial t}\right) e^{c(T-t)} \psi_{\alpha}(x)\left(\frac{\partial^{2} v_{\alpha}}{\partial x^{2}}\right)^{2} d x d t .
\end{aligned}
$$

Take $a c \geq \partial a / \partial t$. Then, by using identities (24) and (25), from (23), we obtain the inequality

$$
\int_{0}^{T} e^{c(T-t)} \psi_{\alpha}(x)\left(\frac{\partial^{2} v_{\alpha}}{\partial x \partial t}\right)^{2} d x d t \leq 0
$$

which implies that $v_{\alpha} \equiv 0$. By virtue of $(22)$, we conclude that $g \equiv 0$. Thus, there exists a strong generalized solution of problem (9), (10), and inequality (18) holds.

\section{A PRIORI ESTIMATES FOR THE DIFFERENCES $u_{\alpha}-u_{0}$ AND $u_{\alpha}-u_{1}$}

By $u(x, t)$ we denote either the solution $u_{0}$ of problem $(3)$, (4) or the solution $u_{1}$ of problem (5), (6). Let us introduce the new function

$$
v(x, t)=u(x, t)-x \frac{u(1, t)-u(\alpha, t)}{1-\alpha} .
$$


A PRIORI ESTIMATES AND CONTINUOUS DEPENDENCE OF SOLUTIONS

The function $v$ is a strong generalized solution of the problem

$$
\begin{aligned}
\frac{1}{a} \frac{\partial v}{\partial t}-\frac{\partial^{2} v}{\partial x^{2}} & =f(x, t)-\frac{x}{a(1-\alpha)}\left[\frac{\partial u(1, t)}{\partial t}-\frac{\partial u(\alpha, t)}{\partial t}\right], \\
v(x, 0) & =\varphi(x)-x \frac{u(1,0)-u(\alpha, 0)}{1-\alpha}, \\
v(0, t) & =0, \quad \frac{v(1, t)-v(\alpha, t)}{1-\alpha}=0,
\end{aligned}
$$

where $\varphi(x)$ is one of the functions $\varphi_{0}(x)$ and $\varphi_{1}(x)$, depending on the problem to be considered. The difference $w_{\alpha}=v-v_{\alpha}$ is a strong generalized solution of the problem

$$
\begin{aligned}
\frac{1}{a} \frac{\partial w_{\alpha}}{\partial t}-\frac{\partial^{2} w_{\alpha}}{\partial x^{2}} & =\frac{x}{a}\left[h^{\prime}(t)-\frac{\left.\frac{\partial u(1, t)}{\partial t}-\frac{\partial u(\alpha, t)}{\partial t}\right]}{1-\alpha}\right], \\
w_{\alpha}(x, 0) & =\varphi-\varphi_{\alpha}-x\left[\frac{u(1,0)-u(\alpha, 0)}{1-\alpha}-h(0)\right], \\
w_{\alpha}(0, t) & =0, \quad \frac{w_{\alpha}(1, t)-w_{\alpha}(\alpha, t)}{1-\alpha}=0,
\end{aligned}
$$

and $w_{\alpha}$ satisfies the inequality [see (11)]

$$
\begin{aligned}
\sup _{0 \leq t \leq T} & \int_{0}^{1} \psi_{\alpha}(x) w_{\alpha}^{2}(x, t) d x+\int_{0}^{T} \int_{0}^{1} \psi_{\alpha}(x)\left(\frac{\partial w_{\alpha}(x, t)}{\partial x}\right)^{2} d x d t \\
\leq & 2 C\left(\int_{0}^{1} \psi_{\alpha}(x)\left|\varphi(x)-\varphi_{\alpha}(x)\right|^{2} d x+\int_{0}^{1} \psi_{\alpha}(x) x^{2}\left|\frac{u(1,0)-u(\alpha, 0)}{1-\alpha}-h(0)\right|^{2} d x\right. \\
& \left.+\int_{0}^{T} \int_{0}^{1} \psi_{\alpha} \frac{x^{2}}{a^{2}(x, t)}\left|h^{\prime}(t)-\frac{\frac{\partial u(1, t)}{\partial t}-\frac{\partial u(\alpha, t)}{\partial t}}{1-\alpha}\right|^{2} d x d t\right) .
\end{aligned}
$$

Inequality (27) remains valid if we use the inequality $\psi_{\alpha}(x) \geq 1-x$ on its left-hand side and the inequality $\psi_{\alpha}(x) \leq 1$ on the right-hand side. We apply the resulting inequality to the difference

$$
u-u_{\alpha}=w_{\alpha}+x\left[\frac{u(1, t)-u(\alpha, t)}{1-\alpha}-h(t)\right],
$$

where $u(x, t)$ is either the solution $u_{0}$ of problem (3), (4) or the solution $u_{1}$ of problem (5), (6). As a result, we obtain the a priori estimate

$$
\begin{aligned}
\sup _{0 \leq t \leq T} \int_{0}^{1}(1-x)\left|u_{0}-u_{\alpha}\right|^{2} d x+\int_{0}^{T} \int_{0}^{1}(1-x)\left|\frac{\partial u_{0}}{\partial x}-\frac{\partial u_{\alpha}}{\partial x}\right|^{2} d x d t \\
\leq C\left\{\int_{0}^{1}\left|\varphi_{0}(x)-\varphi_{\alpha}(x)\right|^{2} d x+\int_{0}^{T}\left[\left|\frac{\alpha h(t)-u_{0}(\alpha, t)}{1-\alpha}\right|^{2}+\left|\frac{\alpha h^{\prime}(t)-\frac{\partial u_{0}(\alpha, t)}{\partial t}}{1-\alpha}\right|^{2}\right] d t\right. \\
\left.+\left|\frac{\alpha h(0)-\varphi_{0}(\alpha)}{1-\alpha}\right|^{2}\right\}
\end{aligned}
$$


where $u_{0}$ is the solution of problem (3), (4), and the a priori estimate

$$
\begin{aligned}
& \sup _{0 \leq t \leq T} \int_{0}^{1}(1-x)\left|u_{1}-u_{\alpha}\right|^{2} d x+\int_{0}^{T} \int_{0}^{1}(1-x)\left|\frac{\partial u_{1}}{\partial x}-\frac{\partial u_{\alpha}}{\partial x}\right|^{2} d x d t \\
& \leq C\left\{\int_{0}^{1}\left|\varphi_{1}(x)-\varphi_{\alpha}(x)\right|^{2} d x+\int_{0}^{T}\left[\left|\frac{u_{1}(1, t)-u_{1}(\alpha, t)}{1-\alpha}-h(t)\right|^{2}\right.\right. \\
&\left.\left.+\left|\frac{\frac{\partial u_{1}(1, t)}{\partial t}-\frac{\partial u_{1}(\alpha, t)}{\partial t}}{1-\alpha}-h^{\prime}(t)\right|^{2}\right] d t+\left|\frac{\varphi_{1}(1)-\varphi_{1}(\alpha)}{1-\alpha}-h(0)\right|^{2}\right\},
\end{aligned}
$$

where $u_{1}$ is the solution of problem (5), (6). Note that the constants $C$ in inequalities (11) and (27)-(29) are in general different but independent of the parameter $\alpha$, the solutions $u_{0}, u_{\alpha}$, and $u_{1}$, the initial data of the problems, the coefficient $a$, the right-hand side $f$ of the equations, and the function $h$.

\section{CONTINUOUS DEPENDENCE OF SOLUTIONS OF PROBLEM (1), (2) ON THE PARAMETER $\alpha$}

Theorem 2. If

$$
\lim _{\alpha \rightarrow 0} \int_{0}^{T}\left|\varphi_{\alpha}(x)-\varphi_{0}(x)\right|^{2} d x=0
$$

then

$$
\lim _{\alpha \rightarrow 0}\left[\sup _{0 \leq t \leq T} \int_{0}^{1}(1-x)\left|u_{\alpha}-u_{0}\right|^{2} d x+\int_{0}^{T} \int_{0}^{1}(1-x)\left|\frac{\partial u_{\alpha}}{\partial x}-\frac{\partial u_{0}}{\partial x}\right|^{2} d x d t\right]=0 .
$$

Proof. Since the solutions of problem (3), (4) satisfy the relations

$$
\lim _{\alpha \rightarrow 0} \int_{0}^{T} u_{0}(\alpha, t) d t=0, \quad \lim _{\alpha \rightarrow 0} \int_{0}^{T}\left|\frac{\partial u_{0}(\alpha, t)}{\partial t}\right|^{2} d x=0
$$

and $\lim _{\alpha \rightarrow 0} \varphi_{0}(\alpha)=0$, it follows from (30) and inequality (28) that relation (31) holds.

To complete the considerations, it remains to show that, for each function $\varphi_{0} \in W_{2}^{1}(0, T)$ satisfying the conditions $\varphi_{0}(0)=0$ and $\varphi_{0}(1)=h(0)$, there exist functions $\varphi_{\alpha} \in W_{2}^{1}(0, T)$ such that $\varphi_{\alpha}(0)=0,\left(\varphi_{\alpha}(1)-\varphi_{\alpha}(\alpha)\right) /(1-\alpha)=h(0)$, and relation (30) is valid. Obviously, one can take $\varphi_{\alpha}(x)=\varphi_{0}(x)-x\left(\left(\alpha h(0)-\varphi_{0}(\alpha)\right) /(1-\alpha)\right)$. The proof of Theorem 2 is complete.

Theorem 3. If

$$
\lim _{\alpha \rightarrow 1} \int_{0}^{1}\left|\varphi_{\alpha}(x)-\varphi_{1}(x)\right|^{2} d x=0
$$

then

$$
\lim _{\alpha \rightarrow 0}\left[\sup _{0 \leq t \leq T} \int_{0}^{1}(1-x)\left|u_{\alpha}-u_{1}\right|^{2} d x+\int_{0}^{T} \int_{0}^{1}(1-x)\left|\frac{\partial u_{\alpha}}{\partial x}-\frac{\partial u_{1}}{\partial x}\right|^{2} d x d t\right]=0 .
$$


Proof. Since the solutions of problem (5), (6) satisfy the relation

$$
\lim _{\alpha \rightarrow 1} \int_{0}^{T}\left[\left|\frac{u_{1}(1, t)-u_{1}(\alpha, t)}{1-\alpha}-h(t)\right|^{2}+\left|\frac{\frac{\partial u_{1}(1, t)}{\partial t}-\frac{\partial u_{1}(\alpha, t)}{\partial t}}{1-\alpha}-h^{\prime}(t)\right|^{2}\right] d t=0
$$

and $\lim _{\alpha \rightarrow 1} \frac{\varphi_{1}(x)-\varphi_{1}(\alpha)}{1-\alpha}=h(0)$, it follows from condition (32) and inequality (29) that relation (31) is valid. To complete the considerations, it remains to show that, for each function $\varphi_{1} \in W_{2}^{1}(0, T)$ satisfying the conditions $\varphi_{1}(0)=0$ and $\varphi_{1}^{\prime}(1)=h(0)$, there exist functions $\varphi_{\alpha} \in W_{2}^{1}(0, T)$ such that $\varphi_{\alpha}(0)=0,\left(\varphi_{\alpha}(1)-\varphi_{\alpha}(\alpha)\right) /(1-\alpha)=h(0)$, and relation (32) holds. Indeed, one can take

$$
\varphi_{\alpha}(x)=\varphi_{1}(x)-x\left(\frac{\varphi_{1}(1)-\varphi_{1}(\alpha)}{1-\alpha}-h(0)\right) .
$$

The proof of Theorem 3 is complete.

\section{REFERENCES}

1. Yurchuk N.I., in Analytic Methods of Analysis and Differential Equations: AMADE, 2003, Cottenham: Cambridge, 2005, pp. 243-250.

2. Kamynin, L.I., Differ. Uravn., 1972, vol. 8, no. 6, pp. 1015-1026.

3. Ionkin, N.I., Differ. Uravn., 1977, vol. 13, no. 2, pp. 294-304.

4. Ionkin, N.I., Differ. Uravn., 1979, vol. 15, no. 7, pp. 1279-1283.

5. Ionkin, N.I. and Moiseev, E.I., Differ. Uravn., 1979, vol. 15, no. 7, pp. 1284-1295.

6. Yurchuk, N.I., Differ. Uravn., 1986, vol. 22, no. 12, pp. 2117-2126.

7. Kartynnik, A.V., Differ. Uravn., 1990, vol. 26, no. 2, pp. 1568-1575.

8. Maurin, K., Metody przestrzeni Hilberta (Methods of Hilbert Spaces), Warszawa: PWN, 1959. Translated under the title Metody gil'bertova prostranstva, Moscow, 1965. 